\title{
Prevalência de retenção escolar e fatores associados em adolescentes da coorte de nascimentos de 1993 em Pelotas, Brasil
}

\author{
Maria de Fátima A. Vieira, ${ }^{1}$ Alicia Matijasevich, ${ }^{2}$ Magda F. Damiani, ${ }^{3}$ \\ Samanta W. Madruga, ${ }^{2}$ Marilda B. Neutzling, ${ }^{2}$ Ana M. B. Menezes, ${ }^{2}$ \\ Cora L. Araújoº e Pedro C. Hallal²
}

Como citar Vieira MFA, Matijasevich A, Damiani MF, Madruga SW, Neutzling MB, Menezes AMB, et al. Prevalência de retenção escolar e fatores associados em adolescentes da coorte de nascimentos de $1993 \mathrm{em}$ Pelotas, Brasil. Rev Panam Salud Publica. 2012;31(4):303-9.

RESUMO Objetivo. Avaliar a ocorrência de retenção escolar até os 11 anos de idade e os fatores associados à retenção.

Métodos. Estudo prospectivo, incluindo 4452 adolescentes da coorte de nascidos em Pelotas, Estado do Rio Grande do Sul, Brasil, em 1993. A amostra representa 87,5\% da coorte original. A retenção escolar foi definida como a repetição de pelo menos uma série escolar até a data da entrevista. As variáveis independentes analisadas foram: sexo, cor da pele, peso ao nascer, indice de bens, idade e escolaridade materna, tipo de escola (privada, estadual ou municipal), idade de ingresso na escola e trabalho.

Resultados. A frequência de retenção escolar foi de 36,3\%, sendo de $42,8 \%$ entre os meninos e 30,0\% entre as meninas. Na análise ajustada, quanto menor a escolaridade da mãe, $o$ indice de bens e o peso ao nascer, maior foi o risco de retenção escolar em ambos os sexos. Adolescentes cuja cor da pele era parda/preta, aqueles que frequentavam escolas públicas e aqueles que ingressaram na escola com 7 anos ou mais apresentaram maior risco de retenção escolar. Apenas entre os meninos, o trabalho infantil esteve associado com a ocorrência de retenção. Conclusões. O baixo nível socioeconômico e a baixa escolaridade materna foram os fatores mais fortemente associados com a retenção escolar. Estratégias para a redução desse evento devem levar em consideração características demográficas e socioeconômicas.

Palavras-chave

Baixo rendimento escolar; fatores socioeconômicos; adolescente; estudos de coortes; Brasil.

Apesar das modificações pelas quais o sistema escolar brasileiro vem passando, como, por exemplo, a ampliação do ensino fundamental de 8 para 9 anos, a educação no país continua enfrentando problemas como retenção escolar e dis-

\footnotetext{
1 Universidade Federal de Pelotas (UFPel), Programa de Pós-Graduação em Nutrição e Alimentos, Pelotas (RS), Brasil. Correspondência: fvieira.nut@gmail.com

2 UFPel, Programa de Pós-Graduação em Epidemiologia, Pelotas (RS), Brasil.

3 UFPel, Programa de Pós-Graduação em Educação, Pelotas (RS), Brasil.
}

torção série-idade. Dados da Diretoria de Estatísticas Educacionais do Instituto Nacional de Estudos e Pesquisas Educacionais Anísio Teixeira (INEP), do Ministério da Educação, revelam que, em 2010, a distorção série-idade em escolas urbanas para o Brasil como um todo foi de $21,3 \%$ no ensino fundamental e $34,2 \%$ no ensino médio. Tais percentuais são ligeiramente superiores no Rio Grande de Sul (22,4 e 30,5\%, respectivamente) e consideravelmente maiores em Pelotas, cidade onde esta investigação foi realizada: 31,9 e $38,2 \%$. A distorção série-idade indica os percentuais de crianças e adolescentes que estão em série/ano inadequado (inferior) para sua idade, sendo, portanto, importante entender os fatores associados à retenção escolar para que se possa ter subsídios para combatê-la (1).

Pesquisas sobre insucesso escolar vêm sendo realizadas sistematicamente no Brasil desde a década de 1930 (2-6). No entanto, a retenção escolar continua a assombrar o sistema educacional, como 
mostram os dados apresentados. Sabe-se que a reprovação repetida usualmente resulta em exclusão do aluno, não só da escola, mas também da sociedade, principalmente entre as classes populares, acarretando desperdícios de recursos e vagas escolares, além de desgaste pessoal para estudantes e professores (7).

Assim sendo, é importante que esse problema seja constantemente monitorado e estudado, de modo que novas estratégias para combatê-lo possam ser definidas. Os estudos sobre as causas da retenção e de outros indicadores do insucesso escolar voltam-se em geral para dois focos: o contexto extraescolar, centrado em variáveis biológicas, psicológicas ou sociais/culturais relativas ao aluno e sua família; e o contexto intraescolar, centrado em aspectos institucionais, que consideram que a própria escola é responsável pelo insucesso por não estar preparada para atender as classes populares, onde o fenômeno se mostra mais prevalente. As duas instâncias devem ser consideradas, na medida em que enfocam esse problema extremamente complexo de diferentes pontos de vista.

O objetivo do presente estudo foi identificar a frequência da retenção escolar entre os adolescentes que compõem a coorte de nascimentos de 1993 da Cidade de Pelotas e avaliar o efeito de fatores demográficos e socioeconômicos e do peso ao nascer sobre esse fenômeno.

\section{MÉTODO}

O presente estudo foi realizado com adolescentes pertencentes à coorte de nascidos vivos no ano de 1993, na Cidade de Pelotas, Brasil. A cidade, que possui cerca de 330 mil habitantes, de acordo com os dados do Censo Demográfico de 2010, fica localizada na região sul do Estado do Rio Grande do Sul, distante $250 \mathrm{~km}$ da capital Porto Alegre. Onze anos após o nascimento, 87,5\% dos nascidos naquele ano foram novamente entrevistados e avaliados em relação à situação nutricional e de saúde em geral, além das condições socioeconômicas e comportamentais. A metodologia e os principais resultados do estudo perinatal e do acompanhamento aos 11 anos estão publicados em artigos anteriores $(8,9)$.

Considerou-se retenção escolar a ocorrência de repetição de pelo menos uma série da escola regular até o momento da entrevista, aos 11 anos de idade. As variáveis independentes avaliadas foram: sexo, cor da pele (autorreferida), escolaridade materna (anos completos), índice de bens (dividido em quintis, do mais pobre para o mais rico), peso de nascimento (categorizado em $<2500 \mathrm{~g}$, 2500 até 3499 g e $\geq 3500$ g), tipo de escola (particular, estadual ou municipal), idade de ingresso na escola (menos de 7 anos e 7 ou mais anos de idade) e ter tido algum trabalho remunerado no período de 2004 a 2005. Nas categorias de cor da pele, pele preta e pele parda foram agrupadas como pele preta/parda devido ao pequeno número de indivíduos que declararam ter cor da pele parda. A variável índice de bens baseou-se na análise de componentes principais, considerando a posse de utilidades domésticas (televisão colorida, geladeira, rádio, som, máquina de lavar roupas, carro, empregada doméstica, acesso à Internet, número de banheiros) e características do domicílio (10).

Foi realizada dupla digitação dos dados no programa Epi Info 6.0, com checagem automática de consistência e amplitude. As análises foram realizadas no pacote estatístico Stata (Stata Statistical Software: Release 9.2. College Station, TX: Stata Corporation; 2003) seguindo modelo hierarquizado de análise. As variáveis do primeiro nível (mais distal) foram escolaridade e idade da mãe, índice de bens e cor da pele. No segundo nível, foi incluído o peso ao nascer. Já o terceiro e último nível (mais proximal) incluiu as variáveis tipo de escola, idade de ingresso na escola e trabalho em 2004 a 2005. As análises brutas e ajustadas foram realizadas usando regressão de Poisson com ajuste robusto da variância (11).

A pesquisa foi aprovada pelo Comitê de Ética em Pesquisa da Universidade Federal de Pelotas, e a participação dos adolescentes foi autorizada por escrito pelos pais ou responsáveis.

\section{RESULTADOS}

Em 2004 e 2005 foram entrevistados 4452 jovens pertencentes à coorte de nascidos vivos na Cidade de Pelotas em 1993. Dados sobre a frequência escolar foram obtidos de 4426 indivíduos, dos quais 4372 estudavam em escolas regulares, 25 em escolas especiais (frequentadas por pessoas com necessidades educativas específicas) e 26 tanto em escolas especiais como em escolas regulares. Apenas três adolescentes não frequentaram nenhum tipo de escola em 2004 e 2005.

A tabela 1 apresenta as prevalências de retenção escolar na população estudada segundo características demográficas e socioeconômicas. A retenção escolar foi de $36,3 \%$ na amostra total e de $42,8 \%$ entre adolescentes do sexo masculino.

Considerando o conjunto de todos os adolescentes estudados (tabela 1), as prevalências de retenção foram superiores a $50 \%$ entre aqueles de cor da pele preta/parda, que pertenciam a famílias mais pobres, naqueles cujas mães tinham menor nível de escolaridade e nos jovens que trabalharam em 2004 e 2005. Foram também altas, acima de $45 \%$, as prevalências de retenção entre os adolescentes que nasceram com baixo peso, naqueles cujas famílias foram classificadas no segundo quintil do índice de bens, nos filhos de mães com menos de 20 anos de idade e naqueles que ingressaram na escola com mais de 7 anos de idade.

A tabela 2 mostra a associação bruta e ajustada entre repetência escolar e as variáveis independentes para o sexo masculino. Com relação às variáveis do primeiro nível, o índice de bens e a escolaridade materna mostraram forte tendência linear negativa com o desfecho, tanto na análise bruta quanto na ajustada: quanto menor o índice de bens da família e a escolaridade materna, maior o risco de retenção escolar. A cor da pele mostrou-se associada ao desfecho, sendo os jovens de cor da pele preta ou parda aqueles que apresentaram maior risco para a retenção.

O baixo peso ao nascer também se mostrou associado à retenção escolar entre os adolescentes do sexo masculino, com tendência linear negativa, ou seja, quanto menor o peso ao nascer, maior o risco de retenção. Com relação à idade materna, após ajuste para as variáveis de confusão, a associação não mostrou significância estatística. Outro fator de risco para retenção escolar foi estudar em escolas estaduais ou municipais. Associação no mesmo sentido foi observada entre aqueles que iniciaram os estudos após os 7 anos de idade, e ainda entre adolescentes que trabalharam em 2004 e 2005.

A tabela 3 mostra a associação bruta e ajustada entre retenção escolar e as variáveis independentes entre as meninas. Índice de bens, escolaridade e idade da mãe, cor da pele, peso ao nascer, tipo de escola e idade de ingresso na escola apre- 
TABELA 1. Retenção escolar conforme variáveis demográficas, socioeconômicas, nutricionais e escolares na coorte de 1993, Pelotas (RS), Brasil, acompanhamento de 2004 a 2005

\begin{tabular}{|c|c|c|c|c|c|c|}
\hline \multirow[b]{2}{*}{ Variável } & \multicolumn{2}{|r|}{ Total } & \multicolumn{2}{|c|}{ Meninos } & \multicolumn{2}{|c|}{ Meninas } \\
\hline & No. & $\begin{array}{l}\% \text { de } \\
\text { retenção }\end{array}$ & No. & $\begin{array}{c}\text { \% de } \\
\text { retenção }\end{array}$ & No. & $\begin{array}{l}\text { \% de } \\
\text { retenção }\end{array}$ \\
\hline Cor da pele (autorreferida) & 4372 & $\begin{array}{c}36,3 \\
P<0,001^{a}\end{array}$ & 2144 & $\begin{array}{c}42,8 \\
P<0,001^{a}\end{array}$ & 2228 & $\begin{array}{c}30,0 \\
P<0,001^{a}\end{array}$ \\
\hline Branca & 2866 & 29,0 & 1432 & 34,7 & 1434 & 23,4 \\
\hline Preta/parda & 1212 & 60,0 & 563 & 59,5 & 649 & 43,6 \\
\hline Outra & 193 & 40,4 & 91 & 48,3 & 102 & 33,3 \\
\hline Peso ao nascer (gramas) & & $P<0,001^{b}$ & & $P<0,001^{b}$ & & $P<0,001^{b}$ \\
\hline$\geq 3500$ & 1129 & 29,7 & 643 & 34,5 & 486 & 23,2 \\
\hline 2500 a 3499 & 2773 & 36,9 & 1296 & 43,8 & 1477 & 30,7 \\
\hline$<2500$ & 379 & 47,2 & 154 & 59,1 & 225 & 39,1 \\
\hline Índice de bens (quintis) ${ }^{c}$ & & $P<0,001^{b}$ & & $P<0,001^{b}$ & & $P<0,001^{b}$ \\
\hline $1^{0}$ & 819 & 60,3 & 418 & 67,2 & 401 & 53,1 \\
\hline $2^{\circ}$ & 824 & 46,6 & 398 & 51,3 & 426 & 42,2 \\
\hline $3^{\circ}$ & 836 & 33,0 & 387 & 43,7 & 449 & 23,8 \\
\hline $4^{\circ}$ & 830 & 24,5 & 401 & 30,4 & 429 & 18,9 \\
\hline $5^{\circ}$ & 843 & 10,4 & 428 & 14,5 & 415 & 6,3 \\
\hline Idade da mãe (anos) & & $P<0,001^{b}$ & & $P=0,001^{b}$ & & $P<0,001^{b}$ \\
\hline$>35$ & 475 & 45,1 & 380 & 50,3 & 242 & 23,1 \\
\hline 20 a 35 & 3066 & 34,5 & 1483 & 40,8 & 1583 & 28,6 \\
\hline$<20$ & 746 & 30,1 & 233 & 37,3 & 366 & 39,9 \\
\hline Escolaridade da mãe (anos) & & $P<0,001^{b}$ & & $P<0,001^{b}$ & & $P<0,001^{b}$ \\
\hline 0 a 4 & 1102 & 58,9 & 542 & 66,9 & 560 & 51,1 \\
\hline 5 a 8 & 1839 & 36,8 & 880 & 44,5 & 959 & 29,7 \\
\hline 9 a 11 & 921 & 20,1 & 461 & 23,4 & 460 & 16,7 \\
\hline$\geq 12$ & 414 & 5,6 & 207 & 7,7 & 207 & 3,4 \\
\hline Tipo de escola & & $P<0,001^{b}$ & & $P<0,001^{b}$ & & $P<0,001^{b}$ \\
\hline Particular & 465 & 13,7 & 233 & 15,0 & 234 & 12,4 \\
\hline Estadual & 1901 & 33,9 & 894 & 40,7 & 1007 & 27,8 \\
\hline Municipal & 1894 & 43,0 & 954 & 49,6 & 940 & 36,3 \\
\hline Idade de ingresso na escola & & $P<0,001^{a}$ & & $P<0,001^{a}$ & & $P<0,001^{a}$ \\
\hline$<7$ anos & 2334 & 27,6 & 1112 & 33,1 & 1222 & 22,5 \\
\hline$\geq 7$ anos & 1953 & 45,8 & 984 & 52,2 & 969 & 39,2 \\
\hline Trabalho em 2004 e 2005 & & $P<0,001^{a}$ & & $P<0,001^{a}$ & & $P<0,001^{a}$ \\
\hline Não & 4093 & 35,1 & 1972 & 63,6 & 2121 & 29,8 \\
\hline Sim & 187 & 51,9 & 118 & 40,8 & 69 & 31,9 \\
\hline
\end{tabular}

a Teste do $\chi^{2}$ de Pearson.

Teste do $\chi^{2}$ de tendência linear.

c Variável com menor número de observações $(n=4$ 152).

sentaram comportamento semelhante ao observado entre os adolescentes do sexo masculino. Entretanto, a magnitude do efeito do índice de bens sobre a retenção escolar foi maior entre as meninas. Entre as adolescentes do sexo feminino, ter trabalhado em 2004 e 2005 não se mostrou associado à retenção escolar.

As prevalências de retenção escolar entre os sexos segundo o índice de bens evidenciam a associação inversa entre nível socioeconômico e retenção escolar. Cabe destacar que, entre os mais ricos, ou seja, aqueles com maior posse de bens, a prevalência de retenção nos meninos chega a ser o dobro daquela encontrada entre as meninas.

\section{DISCUSSÃO}

A prevalência de retenção escolar encontrada no presente estudo foi muito similar à encontrada por Malta et al. (12) entre alunos de primeira série em escolas de Belo Horizonte (34\%). No entanto, o referido estudo avaliou apenas estudantes ingressantes, ao passo que o presente estudo avaliou estudantes que, em sua maioria, já frequentavam a escola há 4 anos. Na coorte de nascidos em Pelotas no ano de 1982, Damiani (13) relatou uma prevalência de fracasso escolar de $27 \%$ até os 9 anos de idade. Em tal estudo, o fracasso escolar foi definido como pelo menos um episódio de reprovação ou evasão durante a história escolar das crianças.

No estudo da coorte de 1982 (13) e em outros estudos $(3,12)$, o fracasso escolar também foi mais frequente entre os meninos do que entre as meninas, achado que também foi observado em nossa amostra. Segundo Carvalho et al. (14), uma das explicações plausíveis para essa constatação poderia ser o trabalho infantil, pois, de acordo com dados da Organização Internacional do Trabalho (OIT), 2,9 milhões de crianças e adolescentes brasileiros com idades entre 5 e 14 anos já trabalham, sendo que dois terços deles são do sexo masculino. Entretanto, segundo os autores, o trabalho das meninas pode estar subestimado, pois as estatísticas sobre trabalho infantil no Brasil são precárias, devido à informalidade ou ilegalidade, e não contemplam o trabalho doméstico (remunerado ou não) realizado pelas meninas. Os autores argumentam ainda que há indicações de que a opção pelo trabalho remunerado muitas vezes resulta da retenção escolar e do fracasso escolar, e não o contrário.

A Constituição Federal do Brasil, de 1988, determina que a idade mínima para trabalhar é de 16 anos, embora admita o trabalho com 14 anos, na condição de aprendiz. Os dados da Rede Interagencial de Informações para a Saúde (RIPSA) avalia que 5,4 milhões de crianças brasileiras entre 5 e 17 anos estão no mercado de trabalho. Ainda segundo a mesma fonte, quanto menor a renda familiar, mais cedo as crianças começam a trabalhar, e quanto mais pobres as famílias, maior a dependência do trabalho infantil (15).

Os estudos sobre a relação entre desempenho escolar e trabalho infantil mostram resultados controversos. Carvalho (16), analisando o Programa de Erradicação do Trabalho Infantil (PETI), implantado no Brasil na década de 1990, aponta que esse programa promoveu avanços nas condições de nutrição e desempenho escolar dos participantes. Por outro lado, uma revisão da literatura (17) concluiu que o trabalho infantil, embora seja prejudicial ao desempenho escolar, também teve efeitos positivos sobre o processo de escolarização de crianças de baixa renda em alguns estudos, por possibilitar a compra de material escolar, por exemplo. Radmann e Damiani (18), igualmente, mostram que o trabalho infantil não afetou o desempenho escolar (retenção) dos adolescentes do Estudo Longitudinal dos Nascidos em Pelotas em 1982, avaliados aos 13 anos de idade. Entretanto, outro estudo (19), realizado 11 anos mais tarde na mesma cidade, indicou que o trabalho aumentou a probabilidade de retenção escolar em adolescentes avaliados aos 11 e 12 anos de idade. 
TABELA 2. Análise bruta e ajustada da retenção escolar entre adolescentes do sexo masculino na coorte de 1993, Pelotas (RS), Brasil, acompanhamento de 2004 a 2005

\begin{tabular}{|c|c|c|c|c|c|}
\hline \multirow[b]{3}{*}{ Nível $^{a}$} & \multirow[b]{3}{*}{ Variável } & \multicolumn{4}{|c|}{ Meninos } \\
\hline & & \multicolumn{2}{|l|}{ Bruta } & \multicolumn{2}{|l|}{ Ajustada } \\
\hline & & RP (IC95\%) & $P$ & RP (IC95\%) & $P$ \\
\hline \multirow[t]{6}{*}{1} & Índice de bens (quintis) & & & & \\
\hline & $5^{\circ}$ & 1,00 & $<0,001^{b}$ & 1,00 & $<0,001^{b}$ \\
\hline & $4^{\circ}$ & $2,10(1,59$ a 2,76$)$ & & $1,39(1,04$ a 1,86$)$ & \\
\hline & $3^{\circ}$ & $3,01(2,33$ a 3,89$)$ & & $1,78(1,34$ a 2,36$)$ & \\
\hline & $2^{\circ}$ & $3,53(2,75$ a 4,54$)$ & & $1,90(1,44$ a 2,52$)$ & \\
\hline & $1^{0}$ & $4,64(3,65$ a 5,89$)$ & & $2,30(1,75$ a 3,03$)$ & \\
\hline \multirow[t]{5}{*}{1} & Escolaridade da mãe (anos) & & & & \\
\hline & $\geq 12$ & 1,00 & $<0,001^{b}$ & 1,00 & $<0,001^{b}$ \\
\hline & 9 a 11 & $3,03(1,84$ a 4,99$)$ & & $2,17(1,29$ a 3,67$)$ & \\
\hline & 5 a 8 & $5,76(3,57$ a 9,28$)$ & & $3,37(2,01$ a 5,65$)$ & \\
\hline & 0 a 4 & $8,66(5,39$ a 13,9$)$ & & $4,30(2,55$ a 7,24$)$ & \\
\hline \multirow[t]{4}{*}{1} & Cor da pele (autorreferida) & & & & \\
\hline & Branca & 1 & $<0,001$ & 1,00 & $<0,001$ \\
\hline & Preta/parda & $1,71(1,55$ a 1,89$)$ & & $1,36(1,23$ a 1,50$)$ & \\
\hline & Outra & $1,39(1,11$ a 1,74$)$ & & $1,15(0,93$ a 1,43$)$ & \\
\hline \multirow[t]{4}{*}{1} & Idade da mãe (anos) & & & & \\
\hline & $>35$ & 1 & $<0,001$ & 1,00 & 0,081 \\
\hline & 20 a 35 & $1,09(0,91$ a 1,30$)$ & & $1,07(0,91$ a 1,25$)$ & \\
\hline & $<20$ & $1,34(1,10$ a 1,63$)$ & & $1,20(1,00$ a 1,43$)$ & \\
\hline \multirow[t]{4}{*}{2} & Peso ao nascer (gramas) & & & & \\
\hline & $\geq 3500$ & 1 & $<0,001^{b}$ & 1 & $<0,001^{b}$ \\
\hline & 2500 a 3499 & $1,26(1,12$ a 1,43$)$ & & $1,13(1,01$ a 1,28$)$ & \\
\hline & $<2500$ & $1,71(1,44$ a 2,02$)$ & & $1,43(1,21$ a 1,69$)$ & \\
\hline \multirow[t]{4}{*}{3} & Tipo de escola & & & & \\
\hline & Particular & 1,00 & $<0,001$ & 1 & 0,028 \\
\hline & Estadual & $2,71(1,97$ a 3,71$)$ & & $1,21(0,88$ a 1,66$)$ & \\
\hline & Municipal & $3,30(2,41$ a 4,50$)$ & & $1,35(0,98$ a 1,85$)$ & \\
\hline \multirow[t]{3}{*}{3} & $\begin{array}{l}\text { Idade de ingresso na escola } \\
\text { (anos) }\end{array}$ & & & & \\
\hline & $<7$ & 1,00 & $<0,001$ & 1,00 & 0,001 \\
\hline & $\geq 7$ & $1,57(1,42$ a 1,74$)$ & & $1,18(1,07$ a 1,31$)$ & \\
\hline \multirow[t]{3}{*}{3} & Trabalho em 2004 e 2005 & & & & \\
\hline & Não & 1 & $<0,001$ & 1 & 0,010 \\
\hline & Sim & $1,55(1,34$ a 1,80$)$ & & $1,21(1,04$ a 1,42$)$ & \\
\hline
\end{tabular}

a Cada variável foi ajustada para as variáveis do mesmo nível de determinação e acima.

b Teste de Wald para tendência linear.

As prevalências de retenção foram maiores entre os meninos em comparação às meninas em todos os quintis socioeconômicos. Outros estudos encontraram associações positivas entre renda e desempenho escolar em Pelotas (13, 20) e no Brasil (6). A baixa escolaridade materna foi fator de risco para repetência neste estudo, um achado que também já foi observado por outros investigadores $(12,13,21)$. No estudo realizado com a coorte de nascidos em 1982, o fracasso escolar foi 2,3 vezes maior entre os filhos de mães menos escolarizadas (13). No presente estudo, o risco de retenção foi quatro vezes maior entre os adolescentes filhos de mães com menor escolaridade ( 0 a 4 anos) do que entre os filhos de mães com 12 ou mais anos de escolaridade. As comparações devem ser interpretadas com cautela, já que os dois estudos consideraram diferentes categorias e intervalos em anos para a idade materna: no primeiro estudo, foram três categorias (0 a 2 anos; 3 a 5 anos; e 6 anos ou mais), enquanto nosso estudo utilizou quatro categorias ( 0 a 4 anos; 5 a 8 anos; 9 a 11 anos; e 12 anos ou mais). Além disso, no intervalo entre as pesquisas, ocorreu aumento dos níveis de escolaridade materna em Pelotas (22).

A cor da pele preta/parda entre os adolescentes de ambos os sexos mostrouse associada à maior chance de repetência, corroborando dados de outros pesquisadores $(3,23)$. Andrade e Dachs (23), analisando o acesso à educação segundo nível de renda e cor/raça, com dados da Pesquisa Nacional por Amostra de Domicílios (PNAD) de 2003, observaram que houve redução da influência da renda e da cor/raça no acesso à escola na faixa etária entre 7 e 10 anos devido à quase universalização do ensino fundamental no país. Ainda assim, cerca de $10 \%$ das crianças pretas, pardas ou indígenas que pertenciam às faixas de renda mais baixas apresentavam escolaridade inadequada. Segundo os autores, o principal determinante da escolaridade adequada foi a renda familiar, mas a cor/raça teve influência em todas as faixas de renda. $\mathrm{O}$ mesmo estudo mostrou que a desigualdade por renda e cor da pele foi cumulativa e aumentava com a progressão escolar, ou seja, a cada nível de progressão escolar em que foi feita a avaliação, os estudantes mais pobres e de cor da pele preta/parda/indígena apresentavam piores escores de adequação da escolaridade.

$\mathrm{O}$ peso ao nascer mostrou-se negativamente associado à repetência escolar, sendo que os nascidos com peso inferior a $2500 \mathrm{~g}$ apresentaram risco $40 \%$ maior do que os que nasceram com peso acima de 3500 g. Estudos têm mostrado que o peso ao nascer está associado à trajetória do desenvolvimento cognitivo (24). Jefferis et al. (25), ao estudarem uma coorte britânica, observaram que, quanto maior era o peso ao nascer, melhor era o desempenho em matemática aos 7 anos de idade. Além disso, o peso ao nascer mostrou-se associado também com maior nível de qualificação aos 33 anos de idade. Outra conclusão do mesmo estudo foi que o ambiente e a estimulação materna praticamente não afetaram o efeito do baixo peso ao nascer sobre o desempenho em matemática entre os indivíduos avaliados. Horta et al. (26), ao avaliarem jovens de 18 anos nascidos em 1982 na cidade de Pelotas, encontraram associação entre desempenho escolar e peso ao nascer. A média de anos de estudo alcançada entre os que nasceram com peso inferior a $2500 \mathrm{~g}$ e superior a $4000 \mathrm{~g}$ foi de 7,5 e 8,0 anos, respectivamente. Na mesma coorte, foi observado que crianças que nasceram com menor peso apresentavam pior desempenho em testes de desenvolvimento mental e psicomotor (26).

O baixo peso ao nascer está fortemente associado com a posição socioeconômica (renda familiar, educação materna, classe social, entre outros indicadores), sendo mais frequente entre as populações mais desfavorecidas (27). Complicações a curto prazo, tais como morte e morbidade, são frequentes entre os recém-nascidos de baixo peso, mas 
TABELA 3. Análise bruta e ajustada da repetência escolar entre adolescentes do sexo feminino na coorte de 1993, Pelotas (RS), Brasil, acompanhamento de 2004 a 2005

\begin{tabular}{|c|c|c|c|c|c|}
\hline \multirow[b]{3}{*}{ Nívela $^{a}$} & \multirow[b]{3}{*}{ Variável } & \multicolumn{4}{|c|}{ Meninas } \\
\hline & & \multicolumn{2}{|l|}{ Bruta } & \multicolumn{2}{|l|}{ Ajustada } \\
\hline & & RP (IC95\%) & $P$ & RP (IC95\%) & $P$ \\
\hline \multirow[t]{6}{*}{1} & Índice de bens (quintis) & & & & \\
\hline & $5^{\circ}$ & 1,00 & $<0,001^{b}$ & 1,00 & $<0,001^{b}$ \\
\hline & $4^{\circ}$ & $3,01(1,97$ a 4,59$)$ & & $1,94(1,25$ a 3,02$)$ & \\
\hline & $3^{\circ}$ & $3,80(2,53$ a 5,71$)$ & & $2,16(1,40$ a 3,32$)$ & \\
\hline & $2^{\circ}$ & $6,74(4,57$ a 9,94$)$ & & $3,64(2,41$ a 5,51$)$ & \\
\hline & 10 & $8,47(5,77$ a 12,44$)$ & & $4,09(2,69$ a 6,20$)$ & \\
\hline \multirow[t]{5}{*}{1} & Escolaridade da mãe (anos) & & & & \\
\hline & $\geq 12$ & 1,00 & $<0,001^{b}$ & 1,00 & $<0,001^{b}$ \\
\hline & 9 a 11 & $4,94(2,32$ a 10,5$)$ & & $2,85(1,29$ a 6,31$)$ & \\
\hline & 5 a 8 & $8,78(4,21$ a 18,3$)$ & & $3,57(1,63$ a 7,82$)$ & \\
\hline & 0 a 4 & $15,10(7,25$ a 31,4$)$ & & $5,21(2,37$ a 11,44$)$ & \\
\hline \multirow[t]{4}{*}{1} & Cor da pele (autorreferida) & & & & \\
\hline & Branca & 1 & $<0,001^{\mathrm{c}}$ & 1,00 & $<0,001^{c}$ \\
\hline & Preta/parda & $1,86(1,63$ a 2,11$)$ & & $1,31(1,15$ a 1,50$)$ & \\
\hline & Outra & $1,42(1,06$ a 1,90$)$ & & $1,08(0,80$ a 1,44$)$ & \\
\hline \multirow[t]{4}{*}{1} & Idade da mãe (anos) & & & & \\
\hline & $>35$ & 1 & $<0,001^{c}$ & 1,00 & $0,053^{c}$ \\
\hline & 20 a 35 & $1,23(0,97$ a 1,57$)$ & & $1,11(0,89$ a 1,39$)$ & \\
\hline & $<20$ & $1,72(1,32$ a 2,23$)$ & & $1,30(1,01$ a 1,67$)$ & \\
\hline \multirow[t]{4}{*}{2} & Peso ao nascer (gramas) & & & & \\
\hline & $\geq 3500$ & 1 & $<0,001^{b}$ & 1 & $0,030^{\mathrm{b}}$ \\
\hline & 2500 a 3499 & $1,32(1,10$ a 1,58$)$ & & $1,18(0,99$ a 1,40$)$ & \\
\hline & $<2500$ & $1,68(1,33$ a 2,11$)$ & & $1,34(1,07$ a 1,68$)$ & \\
\hline \multirow[t]{4}{*}{3} & Tipo de escola & & & & \\
\hline & Particular & 1 & $<0,001^{c}$ & 1 & $0,034^{c}$ \\
\hline & Estadual & $2,24(1,57$ a 3,19$)$ & & $1,14(0,81$ a 1,61$)$ & \\
\hline & Municipal & $2,92(2,06$ a 4,15$)$ & & $1,32(0,94$ a 1,86$)$ & \\
\hline \multirow[t]{3}{*}{3} & $\begin{array}{l}\text { Idade de ingresso na escola } \\
\text { (anos) }\end{array}$ & & & & \\
\hline & $<7$ & 1 & $<0,001^{c}$ & 1 & $<0,001^{c}$ \\
\hline & $\geq 7$ & $1,74(1,52$ a 1,98$)$ & & $1,26(1,10$ a 1,43$)$ & \\
\hline \multirow[t]{3}{*}{3} & Trabalho em 2004 e 2005 & & & & \\
\hline & Não & 1 & 0,729 & 1 & 0,798 \\
\hline & Sim & $1,06(0,75$ a 1,51$)$ & & $0,95(0,65 \mathrm{a} 1,38)$ & \\
\hline
\end{tabular}

a Cada variável foi ajustada para as variáveis do mesmo nível de determinação e acima.

b Teste de Wald para tendência linear.

c Teste de Wald para heterogeneidade.

também já foram descritas complicações a longo prazo, como transtornos de ansiedade, hiperatividade e déficits de atenção, entre outros (28). O dado encontrado em nosso estudo da associação entre repetência escolar e baixo peso ao nascer já foi relatado por outros investigadores, especialmente na faixa de peso $<1500 \mathrm{~g}$ (29).

Quanto ao tipo de escola, os jovens que frequentavam as escolas públicas municipais apresentaram maiores índices de repetência escolar, diferentemente de resultados encontrados para o Estado do Rio Grande do Sul. Segundo dados do Censo Escolar de 2004, a taxa de reprovação na primeira série do ensino fundamental era maior nas escolas estaduais $(19,3 \%)$ do que nas municipais $(16,9 \%)$. Ainda de acordo com o referido Censo, os índices de retenção eram maiores nas escolas estaduais para o ensino fundamental como um todo. A classificação de tipo de escola no presente estudo foi feita segundo o nível administrativo (municipal, estadual ou particular). Portanto, vale considerar dois aspectos: a) algumas das escolas particulares da Cidade de Pelotas são administradas e mantidas por instituições religiosas e atendem populações de baixa renda, ao passo que algumas escolas públicas atendem populações com nível socioeconômico mais elevado; b) o desempenho das escolas do mesmo nível administrativo pode variar conforme a escola $(13,30)$. Soares e Andrade (30), ao avaliarem a qualidade de ensino das escolas de Belo Horizonte, excluindo a influência do nível socioeconômico sobre o desempenho dos alunos, mostraram que algumas escolas, tanto públicas como particulares, têm sucesso em melhorar o desempenho escolar a despeito do nível socioeconômico. O estudo de Damiani (13), que comparou duas escolas públicas administradas pelo mesmo órgão, mostrou que as duas tinham desempenho contrastante, com taxas de fracasso escolar de 10 e $43 \%$.

A comparação entre o desempenho escolar dos integrantes de duas das coortes de Pelotas (1982 e 1993) mostra resultados semelhantes com relação ao sexo, cor da pele, peso ao nascer e escolaridade materna, apesar de os dois estudos apresentarem algumas diferenças na categorização de variáveis e na idade dos indivíduos. O mesmo pode-se dizer da condição socioeconômica, que, embora tenha sido analisada de forma diferente nos dois estudos, mostrou tendência similar: entre os mais ricos (1982) e entre aqueles com o maior índice de bens (1993), as prevalências de retenção e fracasso escolar foram menores do que entre os mais pobres ou com menor índice de bens.

Dentre as limitações do presente estudo, aponta-se a aplicação do questionário apenas à mãe, sem informação sobre os professores e o projeto pedagógico de cada escola. Não se pode descartar também a ocorrência de confusão residual, visto que pode haver outros fatores que não foram coletados ou analisados, como, por exemplo, motivos de retenção ou morbidade infantil (problemas de saúde mental, hospitalizações), que poderiam atuar como confundidores da associação entre tipo de escola e retenção escolar. Outra limitação é o fato de nossa análise não diferenciar retenção em uma série de retenção em várias séries, assim como também o fato de não conhecermos os motivos que levaram à retenção dos adolescentes nessa coorte.

O presente estudo mostrou que a retenção escolar entre os adolescentes de ambos os sexos da coorte de nascimentos de 1993, na Cidade de Pelotas, foi mais prevalente entre os filhos de mulheres de menor escolaridade, pertencentes a famílias com menor índice de bens, nascidos com baixo peso, estudantes de escolas públicas, que haviam ingressado na escola com 7 anos ou mais e classificados como tendo cor da pele preta/parda. O estudo evidenciou ainda que, entre os adolescentes do sexo masculino que trabalhavam fora, a retenção foi maior do que entre os que não trabalhavam.

Finalmente, nossos dados indicam a necessidade de estudos adicionais sobre 
a retenção escolar e sua associação com variáveis socioeconômicas, demográficas e biológicas. Além disso, mostram a importância de políticas públicas específicas para apoiar a permanência na escola e o avanço dos jovens que apresentam maior vulnerabilidade social ou biológica.
Agradecimentos. Estudo financiado por Wellcome Trust (Implications of early life and contemporary exposures on body composition, human capital, mental health and precursors of complex chronic diseases in three Brazilian cohorts, no. 086974/Z/08/Z), Conselho Nacional de Desenvolvimento Científico e Tecnoló- gico $(\mathrm{CNPq})$ e Fundação de Amparo à Pesquisa do Estado de Rio Grande do Sul (FAPERGS). Etapas iniciais foram financiadas pela União Europeia, Programa Nacional para Centros de Excelência (PRONEX/CNPq; Brasil), Pastoral da Criança (Brasil) e Ministério da Saúde do Brasil.

\section{REFERÊNCIAS}

1. Brasil, Ministério da Educação, Instituto Nacional de Estudos e Pesquisas Educacionais Anísio Teixeira. Indicadores educacionais. Disponível em http://portal.inep.gov.br/ indicadores-educacionais/ Acessado em 10 de outubro de 2011.

2. Del Pino M, Porto G. A exclusão escolar na rede pública municipal de ensino: a história continua no século XXI. $30^{\text {a }}$ Conferência Anual da AMPED; 2007; Caxambu, Brasil. Disponível em: http://www.anped.org.br/ reunioes/30ra/trabalhos/GT13-3803-Int. pdf Acessado em 19 de janeiro de 2011.

3. Alves F, Ortigão I, Franco C. Origem social e risco de repetência: interação raça-capital econômico. Cad Pesqui. 2007;37(130):161-80.

4. Brandão $Z$, Baeta $A$, Rocha $A$. Da evasão e repetência no Brasil: a escola em questão. Rio de Janeiro: Achiamé; 1983.

5. Damiani M. Pedagogic discourse and academic failure in southern Brazil. Em: Daniels $\mathrm{H}$, Garner P, editors. World yearbook of education 1999: inclusive education. London: Koogan Page; 1999. Pp. 169-77.

6. Ferrari A. Diagnóstico da escolarização no Brasil. Rev Bras Educ. 1999;12:22-47.

7. Brasil, Ministério da Educação, Instituto Nacional de Estudos e Pesquisas Educacionais Anísio Teixeira. SAEB-2003 Relatório do Sistema Nacional de Avaliação da Educação Básica. Brasília: Ministério da Educação; 2006. Disponível em: http://www.oei.es/quipu/ brasil/RelatorioSaeb2003_1.pdf Acesso em 26 de janeiro de 2012.

8. Araujo CL, Victora CG, Hallal PC, Gigante DP. Breastfeeding and overweight in childhood: evidence from the Pelotas 1993 birth cohort study. Int J Obes (Lond). 2006;30(3):500-6.

9. Victora CG, Araujo CL, Menezes AM, Hallal PC, Vieira Mde F, Neutzling MB, et al. Methodological aspects of the 1993 Pelotas (Brazil) Birth Cohort Study. Rev Saude Publica. 2006;40(1):39-46.

10. Filmer D, Pritchett LH. Estimating wealth effects without expenditure data-or tears: an application to educational enrollments in states of India. Demography. 2001;38(1): 115-32.

11. Barros AJ, Hirakata VN. Alternatives for logistic regression in cross-sectional studies: an empirical comparison of models that directly estimate the prevalence ratio. BMC Med Res Methodol. 2003;3:21.
12. Malta DC, Goulart EMA, Lima e Costa MFF. Nutritional status and socioeconomic factors associated with failure in school: a prospective study of first grade students in Belo Horizonte, Brazil. Cad Saude Publica. 1998;14(1):157-64.

13. Damiani M. Discurso pedagógico e fracasso escolar. Ensaio: Aval Pol Publ Educ. 2006;14(53):457-78.

14. Carvalho M. Sucesso e fracasso escolar: uma questão de gênero. Educ Pesqui. 2003;29(1): 185-93.

15. RIPSA. Rede Interagencial de Informação para a Saúde. Indicadores básicos para a saúde no Brasil: conceitos e aplicações. $2^{\mathrm{a}}$ ed. Brasília: Organização Pan-Americana da Saúde; 2008. Disponível em: http://www. ripsa.org.br/php/level.php?lang=pt\&compo nent $=68 \&$ item $=20$ Acessado em 26 de janeiro de 2012.

16. Carvalho IMM. Algumas lições do programa de erradicação do trabalho infantil. Sao Paulo Perspec. 2004;18(4):50-61. Disponível em: http://www.scielo.br/pdf/spp/v18n4/ a07v18n4.pdf Acessado em 4 de abril de 2010.

17. Kassouf AL. Trabalho infantil: causas e conseqüências [monografia]. Disponível em: http:/ / www.cepea.esalq.usp.br/ pdf/texto.pdf Acessado em 6 de dezembro de 2010.

18. Radmann FTB, Damiani MF. Trabalho infantil e escolarização: dados do estudo longitudinal dos nascidos em 1982, em Pelotas (RS). XIX Congresso de Iniciação Científica, II Mostra Científica; 2010; Pelotas, Brasil. Disponível em: http://www.ufpel.edu.br/cic/2010/cd/ pdf/CH/CH_01030.pdf Acessado em 26 de janeiro de 2012.

19. Bohm MW, Damiani MF. Trabalho infantil e desempenho escolar: estudo longitudinal dos nascidos em 1993, em Pelotas. XX Congresso de Iniciação Científica, III Mostra Científica; 2010; Pelotas, Brasil. Disponível em: http:// www.ufpel.edu.br/cic/2011/anais/pdf/ $\mathrm{CH} / \mathrm{CH}$ _00567.pdf Acessado em 26 de janeiro de 2012.

20. Victora C, Martines J. Fatores sócio-econômicos, estado nutricional e rendimento escolar: um estudo em 500 crianças de primeira série. Cad Pesqui. 1982;41:38-48.

21. Ivanovic DM, Perez HT, Olivares MG, Diaz NS, Leyton BD, Ivanovic RM. Scholastic achievement: a multivariate analysis of nu- tritional, intellectual, socioeconomic, sociocultural, familial, and demographic variables in Chilean school-age children. Nutrition. 2004;20(10):878-89.

22. Tomasi E, Barros FC, Victora CG. Socioeconomic situation and living conditions: comparison of two population-based cohorts from southern Brazil. Cad Saude Publica. 1996;12(Suppl 1):15-9.

23. Andrade C, Dachs J. Acesso à educação por faixas etárias segundo renda e raça/cor. Cad Pesqui. 2007;37(131):399-422.

24. Power C, Jefferis BJ, Manor O, Hertzman C. The influence of birth weight and socioeconomic position on cognitive development: Does the early home and learning environment modify their effects? J Pediatr. 2006;148(1):54-61.

25. Jefferis BJ, Power C, Hertzman C. Birth weight, childhood socioeconomic environment, and cognitive development in the 1958 British birth cohort study. BMJ. 2002;325(7359):1-6.

26. Horta BL, Sibbritt DW, Lima RC, Victora CG. Weight catch-up and achieved schooling at 18 years of age in Brazilian males. Eur J Clin Nutr. 2007;63(3):369-74.

27. Parker JD, Schoendorf KC, Kiely JL. Associations between measures of socioeconomic status and low birth weight, small for gestational age, and premature delivery in the United States. Ann Epidemiol. 1994;4(4):271-8.

28. Brown AS, Susser ES, Lin SP, Neugebauer $\mathrm{R}$, Gorman JM. Increased risk of affective disorders in males after second trimester prenatal exposure to the Dutch hunger winter of 1944-45. Br J Psychiatry. 1995;166:601-6.

29. Klebanov PK, Brooks-Gunn J, McCormick MC. School achievement and failure in very low birth weight children. J Dev Behav Pediatr. 1994;15(4):248-56.

30. Soares J, Andrade R. Nível socioeconômico, qualidade e equidade das escolas de Belo Horizonte. Ensaio: Aval Pol Publ Educ. 2006; 14(50):107-25.

Manuscrito recebido em 16 de maio de 2011. Aceito em versão revisada em 27 de outubro de 2011. 
ABSTRACT Objective. To evaluate the occurrence of grade retention until 11 years of age and the factors associated with retention.

Prevalence of grade retention and associated factors among adolescents from the 1993 Pelotas, Brazil, birth cohort
Methods. This prospective study included 4452 adolescents from the 1993 city of Pelotas birth cohort (state of Rio Grande do Sul, Brazil). This sample represents $87.5 \%$ of the original cohort. Grade retention was defined as the repetition of at least one school grade until the date of the interview. The following independent variables were analyzed: sex, skin color, birth weight, ownership of goods, age, maternal schooling, type of school (private, state, or city), age at school entry, and employment.

Results. The overall frequency of grade retention was $36.3 \%$, vs. $42.8 \%$ for boys and $30.0 \%$ for girls. The adjusted analysis showed that the lower the level of maternal schooling, ownership of goods, and birth weight, the higher the risk of grade retention for both boys and girls. Black/brown adolescents, those studying in public schools, and those who were 7 years of age or older at school entry had a higher risk of grade retention. For boys, childhood labor was associated with grade retention.

Conclusions. Low socioeconomic and low maternal schooling levels were the factors most strongly associated with grade retention. Strategies to reduce this situation must take into account demographic and socioeconomic characteristics.

Key words Underachievement; socioeconomic factors; adolescent; cohort studies; Brazil. 